\title{
The mathematical model simulation of law risk evaluation for intellectual property protection Zoucaixia Zhoubing
}

\author{
Fudan university,shanghai200438, China \\ Shanghai University of Political science and law, shanghai 201701, China \\ Yonyou software co.Itd.shanghai branch,shanghai200030
}

Keywords: Intellectual property protection; law risks; local clustering improvement; weights entropy

\begin{abstract}
Currently, the intellectual property protection gradually present diverse characteristics in which some disputes become norm. There are no effective evaluation models to assess the law risks in the intellectual property protection. This paper proposes an intellectual property law risks evaluation models based on the weighted entropy of the statistical analyzed law factors clustering which integrates the law evaluation risks factors and illegal analysis constrained factors. According to the local clustering areas of the information entropy segmentation law risk factors of the intellectual property constrained factors, the maximum information entropy of the law risks can be obtained while protecting the intellectual property and the law risks will be graded accordingly. The experimental results show that the proposed evaluation model can accurately assess the law risks in intellectual property protection procedures with high practical values.
\end{abstract}

\section{Introduction}

With the increasing awareness of intellectual property protection, the law conflicts caused by the intellectual property are gradually increasing ${ }^{[1]}$. The awareness of intellectual property protection can increase the enterprise management efficiency and profits which improves the competitiveness of enterprises ${ }^{[2-5]}$. This paper proposes an intellectual property law risks evaluation models based on the weighted entropy of the statistical analyzed law factors clustering which integrates the law evaluation risks factors and illegal analysis constrained factors. According to the local clustering areas of the information entropy segmentation law risk factors of the intellectual property constrained factors, the maximum information entropy of the law risks can be obtained while protecting the intellectual property and the law risks will be graded accordingly. The experimental results show that the proposed evaluation model can accurately assess the law risks in intellectual property protection procedures with high practical values.

\section{The optimal area segment of local clustering in the legal risks of intellectual property protection}

\subsection{The construction and classification of the potential legal risk factors}

The violations during intellectual property protection can form an assessment set. By calculating the average entropy of the data in the set, different categories can be classified to obtain the law risks factors under different clustering conditions. The support vector machine approaches can normalize the classification hyper-planes of the intellectual property protection law risks factors. Suppose the parameter $\Delta=1$ in the support vector machine with proper compress coefficients $\mathrm{u}$ and $\mathrm{k}$, the support vector machine can be constrained with following equations.

$$
\begin{aligned}
& u \cdot x+\mathrm{k}=1, \text { if } \mathrm{y}=1 \\
& u \cdot x+\mathrm{k}=-1, \text { if } \mathrm{y}=-1
\end{aligned}
$$

The distance between the category hyper-plane of the law risks factors and the supportive vector is $1 /\|u\|$. The convergence constrained linear programming functions can classify the potential factors, which is described by equation (2). 


$$
\min _{u, k} \frac{1}{2}\|u\|^{2}
$$

z. p. $y_{i}\left(u \cdot x_{i}+k\right) \geq 1, \mathrm{i}=1, \ldots, h$.

The optimal solutions of the convergence constrained conditional target function can be obtained with optimization theory. The Lagrange equation can be expressed by

$$
H=\frac{1}{2}\|u\|^{2}+\sum_{i=1}^{h} \beta_{i}\left[1-y_{i}\left(e \cdot x_{i}+k\right)\right]
$$

In the equation, $\mathrm{y}_{\mathrm{i}}\left(u \cdot x_{i}+k\right) \geq 1$ describes the constrains norms, $\beta_{i} \geq 0$ describes the Lagrange multiplier for the constrains norms. According to KKT Standards, there is

$$
\frac{\partial}{\partial u} H=u-\sum_{i=1}^{h} \beta_{i} y_{i} x_{i}=0 \Rightarrow u=\sum_{i=1}^{h} \beta_{i} y_{i} x_{i} \quad \frac{\partial}{\partial k} H=\sum_{i=1}^{h} \beta_{i} y_{i}=0
$$

Based on Wolf duality theory, the optimal Wolf dual problem can be expressed by

$$
\max _{\alpha} u(\beta)=\sum_{i=1}^{h} \beta_{i}-\frac{1}{2} \sum_{i, j=1}^{h} \beta_{i} \beta_{j} y_{i} y_{j} x_{i} \cdot x_{j}
$$

z. p. $\quad \sum_{i=1}^{h} \beta_{i} y_{i}=0, \beta_{i} \geq 0, \mathrm{i}=1, \ldots, h$.

The optimal solutions of the previous equations can form optimal hyper-plane. If $\beta$ describes the operation norm of the optimal solutions, $\beta$ will be 0 ; if $\beta$ is not 0 , the corresponding vector $x_{i}$ is supportive vector and the obtained linear set of the training sample vector is $u^{\square}=\sum_{i=1}^{h} \beta_{i}^{\square} y_{i} x_{i}$. For arbitrary supportive vector $x_{i}, \quad k^{\square}$ can be determined as $k^{\square}=y_{i}-u^{\square} \cdot x_{i}$. The final analysis function can be expressed by

$g(x)=\log a l\left(u \cdot x+k^{\square}\right)=\log a l\left(\sum_{i=1}^{h} \beta_{i}^{\square} y_{i} x_{i} \cdot x+k^{\square}\right)$

In the formula, $\log a l(\cdot)$ describes the sign function and there is:

$\log a l(p)= \begin{cases}0 & p<0 \\ 1 & p \geq 0\end{cases}$

Equation (6) is used to classify the law risks analysis factors. The corresponding law risks types can be determined according to some certain categories.

\subsection{Illegal grading}

The accurate analysis on the intellectual property protection law risks can rate and segment the risks analysis factors. The 5-point analysis method can analyze the key risk factors meeting customers' evaluation standard. The information entropy is used to study related ranges of the correlative factors. The analysis and rating evaluation results of the local clustering areas of the large scale intellectual property protection are shown in Table 1.

Table 1 The intellectual proper law risks evaluation of some enterprise

\begin{tabular}{llccc}
\hline $\begin{array}{l}\text { Grades } \\
\text { feedbacks }\end{array}$ & Levels & Amounts of patents & Complete time & Law \\
\hline $0-0.6$ & Lost control & $20-40$ & $700-400$ & $0.7-1$ \\
$0.6-1.3$ & Significant risks & $40-50$ & $400-300$ & $0.70-0.80$ \\
$1.3-1.6$ & Moderate risks & $50-67$ & $300-210$ & $0.70-0.50$ \\
$1.6-2.4$ & Common risks & $57-44$ & $170-110$ & $0.50-0.54$ \\
$2.4-3.2$ & The risk past & $40-70$ & $170-140$ & $0.30-0.50$ \\
$3.2-3.8$ & Risks moderate & $90-110$ & $80-70$ & $0.20-0.30$ \\
$3.8-4.2$ & Risks good & $160-1800$ & $70-40$ & $0.08-0.13$ \\
$4.2-5.0$ & Risks excellent & Above 1800 & $40-10$ & Below 0.08
\end{tabular}

The data in Table 1 can be used to analyze the grading of the main parts related factors 
when protect the intellectual property which is diverse and uncertain. Therefore, their proportion weights in the entire law risks analysis model can reflect the change states of the overall risks.

\section{Experimental results and analysis}

In order to verify the validity of the proposed method, some experiments are carried on. The experimental environment is Matlab 7.1 in the platform of Windows XP. The historical data of the intellectual property protection from 5 different types of enterprises are chosen for the research. 1000 pieces of data are collected as the experimental samples. This paper uses SQL methods to collect the data and store them in SQL server databases. This paper emulates and analyzes the law risks changes of some logistics business enterprise during a period of time as shown in Fig. 1.

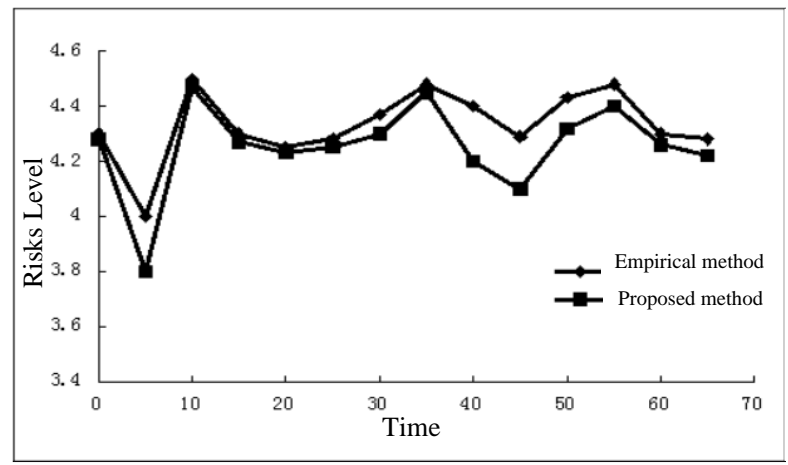

Fig. 1 The comparison of the legal risk assessment models

If the intellectual property rights are stable, the enterprise intellectual property protection risks in some periods should be stable accordingly without significant fluctuations trends. The evaluation curves of the enterprise intellectual property protection law risks with the proposed algorithm can effectively reflect the true states of the logistics enterprise intellectual property protection. The experiments apply 3000 pieces of historical intellectual property data to test the performance of the proposed model as shown in Fig. 2 in which 10 kinds of intellectual property conflicts from 5 different types of enterprises are evaluated by the proposes method for the law risks assessment.

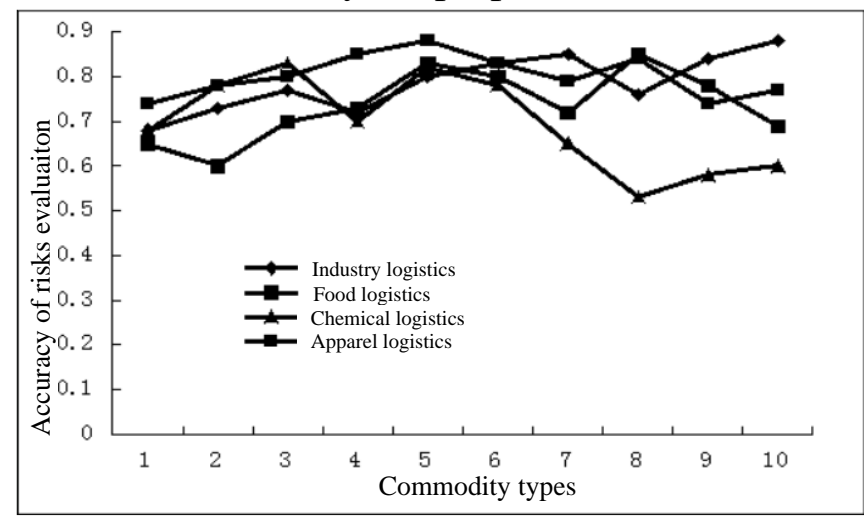

Fig. 2 The comparison of the law risks in intellectual property protection

Fig. 2 describes the intellectual property protection law risks states of different logistics enterprises with the proposed model. The absolute differences between the real logistics law risks and the total amount of logistics volumes are compared. If the amount of the initial logistic law risks is $Q$ and the amount of the logistic intellectual property protection law risks factors after effective research by the measured data is $P$, the accuracy of the transaction risks evaluation after the standard operation by the proposed model is $G=\frac{P}{Q}$.

Figure 3 depicts the different logistics enterprises under state protection of intellectual property legal risks this model, with real legal risks logistics absolute difference and the total amount of logistics comparative results. The number of legal risk analysis if the initial logistics, test data can be effectively developed through the logistics of intellectual property protection laws number of 
risk factors for, so the exact amount of the transaction after a risk assessment by the proposed model for the formation of standardized operation

From the previous analysis, the intellectual property protection law risks in the established $Q$ model are much larger than $75 \%$. The chemical transportation will be badly affected by the features of the raw materials which make the risks analysis substantial fluctuations. The relative risk control capabilities are poor which cannot form stable risk changing curves. It meets the actual logistics situation. The above experimental results illustrate the proposed intellectual property protection law risks evaluation model is consistent with the real situation with satisfactory results.

\section{Conclusions}

This paper proposes an intellectual property law risks evaluation models based on the weighted entropy of the statistical analyzed law factors clustering which integrates the law evaluation risks factors and illegal analysis constrained factors. According to the local clustering areas of the information entropy segmentation law risk factors of the intellectual property constrained factors, the maximum information entropy of the law risks can be obtained while protecting the intellectual property and the law risks will be graded accordingly. The experimental results show that the proposed evaluation model can accurately assess the law risks in intellectual property protection procedures with high practical values.

\section{Project support:}

Young in 2013 the ministry of education of humanities and social science fund project "Chinese character" intellectual property rights TRIPS after the era of cultural reconstruction, based on the nature of intellectual property law philosophy perspectives (13 yjc820120).

Ministry of law under China law society, 2013, the general topic of the ecological theory of "natural rights" - based on the research of ecological law legal basis "(CLS) (2013) C51-1.

\section{References}

[1] JinXiaoHong. electronic commerce credit evaluation model research [D]. Beijing forestry university. 2007.7.82-86.

[2] Fu Shaochuan, jeason, ma jun. Electronic commerce risk analysis and qualitative evaluation method research [J]. Journal of intelligence. 2005. (5) : 18 to 20.

Feng Wen super [3], the risk and prevention countermeasures of e-commerce [D]. Jinan university. 2005.5.34-38.

[4] zhong-hua yan, the risk of enterprise electronic commerce strategy and management countermeasures [J]. Journal of logistics technology. 2004.27 (1) : 039-041.

[5] Liu Dongbiao, group decision making based on the theory of the D S evidence fusion method research [D], shanxi university of finance and economics, 2008.3.5-5. 